\title{
Evaluation of Wound Healing Effect of Punica granatum L Peel Extract on Deep Second-Degree Burns in Rats
}

\author{
Ke $\mathrm{Ma}^{1}$, Mindong $\mathrm{Du}^{2}$, Mingde Liao ${ }^{1 *}$, Shihai Chen ${ }^{1}$, Guoqian Yin ${ }^{1}$, Qingfeng \\ $\mathrm{Liu}^{1}$, Qiang Wei ${ }^{1}$ and Gang Qin ${ }^{2}$ \\ ${ }^{1}$ Department of Plastic \& Cosmetic Surgery, The First Affiliated Hospital of Guangxi Medical University, Nanning, 530021, \\ ${ }^{2}$ Department of Orthopaedics, The First Affiliated Hospital of Guangxi University of Chinese Medicine, Nanning, 530023 \\ Guangxi, China
}

*For correspondence: Email: make133494@163.com; Tel: +8607715356512

Received: 15 August 2014

Revised accepted: 1 December 2014

\begin{abstract}
Purpose: To investigate the healing effect of Punica granatum L. peel (Pomegranate husk) extract (PHE) on deep second-degree burns in rats.

Methods: Male Wistar rats, weighing 220 - 240 g, were subjected to deep second-degree skin burns by electrical scald instrument. The animals were divided into three groups as follows: (1) deep seconddegree burn model (control) group, (2) burns model treated with $1 \%$ silver sulfadiazine (SSD) group, and (3) burns model treated with PHE group. On days 3, 7, 14 and 21, animal weight, wound area as well as histopathological features of skin were evaluated for all the groups. Minimum inhibitory concentration (MIC) of PHE on Staphyloccocus aureus, Pseudomonas aeruginosa and Escherichia coli were also assessed separately.

Results: On day 21, the mean wound area of PHE treatment group $\left(0.21 \pm 0.07 \mathrm{~cm}^{2}\right)$ and $S S D$ treatment group $\left(1.15 \pm 0.1 \mathrm{~cm}^{2}\right)$ were significantly smaller than that of control rats $\left(2.42 \pm 0.2 \mathrm{~cm}^{2}, p<\right.$ $0.01)$ respectively. Histological results indicate that inflammatory cells disappeared substantially and were replaced by new granulation tissue in PHE treatment or SSD treatment group, while that of the control rats still showed severe inflammatory cell infiltration. Antibacterial test revealed that the MIC of PHE on Staphyloccocus aureus, Pseudomonas aeruginosa and Escherichia coli was 6.25, 12.5 and $6.25 \mathrm{mg} \cdot \mathrm{mL}^{-1}$, respectively.

Conclusion: The results reveal that PHE is an effective and promising medicinal herb for the management of deep second degree burns.

Keywords: Punica granatum L. peel, Burns, Wound area, Healing, Antimicrobial activity

Tropical Journal of Pharmaceutical Research is indexed by Science Citation Index (SciSearch), Scopus, International Pharmaceutical Abstract, Chemical Abstracts, Embase, Index Copernicus, EBSCO, African Index Medicus, JournalSeek, Journal Citation Reports/Science Edition, Directory of Open Access Journals (DOAJ), African Journal Online, Bioline International, Open-J-Gate and Pharmacy Abstracts
\end{abstract}

\section{INTRODUCTION}

Every year, millions of people suffer major disability or even death from burns caused by hot water, flame and boiling oil. People suffer from burns due to domestic and industrial accidents, which along with enormous cost of treatment cause mortality and considerable morbidity [1]. According to the World Health Organization
(WHO), there were 300,000 deaths worldwide due to burns in 2012, with $96 \%$ of these deaths occurring in developing countries. Burns are one of the health problems associated with irreparable harm to patients and their families [2].

There are various medicines for burns therapy such as silver sulfadiazine ointment (SSD), mafenide acetate and silver nitrate. SSD is the 
most used topical treatment for burn injury due to its potent antimicrobial efficacy. However, silver gets absorbed systemically, and causes systemic complications such as neutropenia, methemoglobinemia and renal toxicity [3]. Therefore, finding more efficient agents with fewer side effects for treatment of burns has always been a priority for researchers.

Pomegranate husk is obtained from the peel of Punica granatum L., which is rich in tannin, flavones, organic acids and alkaloids. It has pharmacological actions such as astringency, antibacterial and antivirus effect [4]. Pomegranate husk has been used for burn treatment since ancient times in China. The aim of the study was to investigate the healing effect of PHE on deep second-degree burn wounds in rats.

\section{EXPERIMENTAL}

\section{Materials}

The herbal samples of pomegranate husk were collected from Bozhou City, Anhui Province in China in September 2013. Taxonomic identification of the plant was performed by Prof. Le-li Wang of Guangxi University of Chinese Medicine in China. A voucher specimen (NO. PH 201309017) was deposited in the College of Pharmacy, Guangxi University of Chinese Medicine, China for future reference. The powdered sample $(20 \mathrm{~g})$ was placed a round bottom flask with $70 \%$ ethanol $(1: 8, w / v)$ for reflux extraction at $80{ }^{\circ} \mathrm{C}$. It was extracted twice, two hours for each, and then the ethanol was removed by rotary evaporator. The crude extracts was evaporated to dryness, and reconstituted by water. The working concentration of PHE was equivalent to 100 $\mathrm{mg} \cdot \mathrm{mL}^{-1}$ (extract weights / final volume).

\section{Animals and model preparation}

Male Wistar rats weighing 220-240 g were obtained from experimental animal center of Guangxi Province, Guangxi. The animals had free access to feed and water, and were allowed to acclimatize for at least one week before use. All animal experiments were approved by the Animal Care and Use Committee of Guangxi Medical University (approval ref no. 20131007) and was carried out in compliance with Directive 2010/63/EU on the handling of animals used for scientific purposes [5].

After the back hair was removed by a clipper, 20 $\%$ ethyl carbamate solution was used for anesthetizing the rats. The top of electrical scald instrument (manufactured by Changhai Hospital of Second Military Medical University, China) was pressed onto the back skin with a certain force for $15 \mathrm{~s}$ at $75{ }^{\circ} \mathrm{C}$. In this way, the deep second-degree burn model was prepared in rats.

\section{Experimental groups and treatments}

All rats were randomly divided into three groups of thirty-five rats in each: deep second-degree burn model group, SSD-treated group and PHEtreated group. All animals of model, SSD-treated and PHE-treated groups received the deep second-degree burn. SSD cream $(1 \%$, w/w) was used as standard drug.

In a preliminary study, the dose-response properties of PHE and silver sulfadiazine were examined to determine the optimal dose, and the most effective in the wound healing was $0.3 \mathrm{~g}$ SSD or $1 \mathrm{~mL}$ PHE liquid per wound (data not shown).

$1 \mathrm{~mL}$ of PHE liquid or $0.3 \mathrm{~g}$ of SSD was applied slowly with sterile cotton bud to the burn wound area and extended slightly outside the wound area to ensure inclusion of the wound edges. The rats in control group were treated with $1 \mathrm{~mL}$ of saline solution per wound. Treatments were repeated twice daily for 21 days. The first application was done directly after the wound injury. The wound remained exposed after the treatment all through the experiment.

\section{Measurement of body weight and wound area}

On days $0,3,7,14$ and 21 of treatment, body weight and wound area were measured the wounds were photographed with a digital camera in order to calculate wound surface area (WSA)

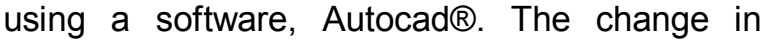
wound surface area in a given day $\left(\mathrm{WSA}_{\mathrm{x}}\right)$ was expressed as a percentage of the wound area on the second day $\left(\mathrm{WSA}_{\mathrm{y}}\right)$ as in Eq 1.

WSA $(\%)=\left\{\left(\mathrm{WSA}_{\mathrm{y}}-\mathrm{WSA}_{\mathrm{x}}\right) / \mathrm{WSA}_{\mathrm{y}}\right\} 100 \ldots \ldots(1)$

\section{Histological study}

Wound skin tissue samples were taken by a scalpel from model, SSD-treated and PHEtreated group rats on days 7, 14 and 21 for histological observation and six rats from each group were sacrificed to obtain skin wound tissues at every time point.

The skin tissues were fixed with $10 \%$ formalin. After fixation, samples were embedded in paraffin, cut into $3 \mathrm{~mm}$ frozen sections with a 
cryostat microtome, then stained with hematoxylin-eosin reagent. Collagen fiber, inflammatory cell, blood vessel, fibroblast and granulation tissue of the rat skin were examined under a microscope separately.

\section{Antibacterial test}

MIC of PHE on Escherichia coli (ATCC25916), Staphyloccocus aureus (ATCC27233) and Pseudomonas aeruginosa (ATCC27845) were determined by agar dilution. The three bacteria were all diluted to $1.5 \times 10^{5} \mathrm{CFU} \cdot \mathrm{mL}^{-1}$ with $0.9 \%$ sodium chloride solution. In a sterilization dilution orifice plate, $3 \mathrm{~mL}$ sterile $0.9 \%$ sodium chloride solution was added into numbered holes. Then 3 $\mathrm{mL}$ PHE was added in first hole, $3 \mathrm{~mL}$ was blended and carried into the second hole, and so forth. At last, the concentrations of diluted PHE were $50,25,12.5,6.25,3.13,1.56,0.78,0.39$ and $0.20 \mathrm{mg} \cdot \mathrm{mL}^{-1}$ in the holes respectively. 1.5 $\mathrm{mL}$ different concentrations of PHE were taken onto sterile plates, and put into agar culturemedium. Plate covers were covered and spinned rapidly. After agar solidified, $2 \mu \mathrm{L}$ bacteria liquid was vaccinated onto them respectively. Together, a negative control (agar without bacterium) and a positive control (agar with bacterium and without drug) were carried out as a control. The plates were put in $37^{\circ} \mathrm{C}$ constant temperature incubator to culture for $48 \mathrm{~h}$, and then the growth of bacterial colony was observed. The experiment was repeated three times to determine MIC.

\section{Statistical analysis}

Values were expressed as means \pm SEM. Multiple group comparisons were performed using one-way analysis of variance (ANOVA) with the SPSS 18.0 followed by Dunnett's test to detect inter-group differences. $P<0.05$ was considered significant in all cases.

\section{RESULTS}

\section{Body weight}

In all the cases, the weight of rats decreased by $3-10 \%$ during the initial period up to 7 days. Thereafter, the weight increased gradually. The mean weight of the SSD treatment group increased more rapidly than control group between days $7(p<0.05)$ and $21(p<0.05)$ while the mean weight of rats in the PHE treatment group decreased more slowly than that of the control group between days $3(p<0.05)$ and $7(p<0.01)$ but increased more rapidly than the control group between days $14(p<0.05)$ and day $21(p<0.05)$ (Figure 1$)$.

\section{Wound healing}

The wound area of deep-second degree burn rats increased in initial 3 days. Thereafter, the wound area decreased progressively. The wounds treated with PHE healed more quickly than those of the control group. The average wound area of PHE treatment group $(0.21 \pm 0.07$ $\mathrm{cm}^{2}$ ) reduced more rapidly than model group $\left(2.42 \pm 0.2 \mathrm{~cm}^{2}\right)$ on day $21(p<0.01)$ (Figure 2). The mean wound area of rats treated with SSD $\left(1.15 \pm 0.1 \mathrm{~cm}^{2}\right)$ was significantly smaller than that of the model group $\left(2.42 \pm 0.2 \mathrm{~cm}^{2}\right)$ on day $21(p<0.01)$. On the 21st day, the mean contraction of PHE-treated wounds was about 97 $\%$, whereas that of the wounds treated with SSD was $79 \%$.

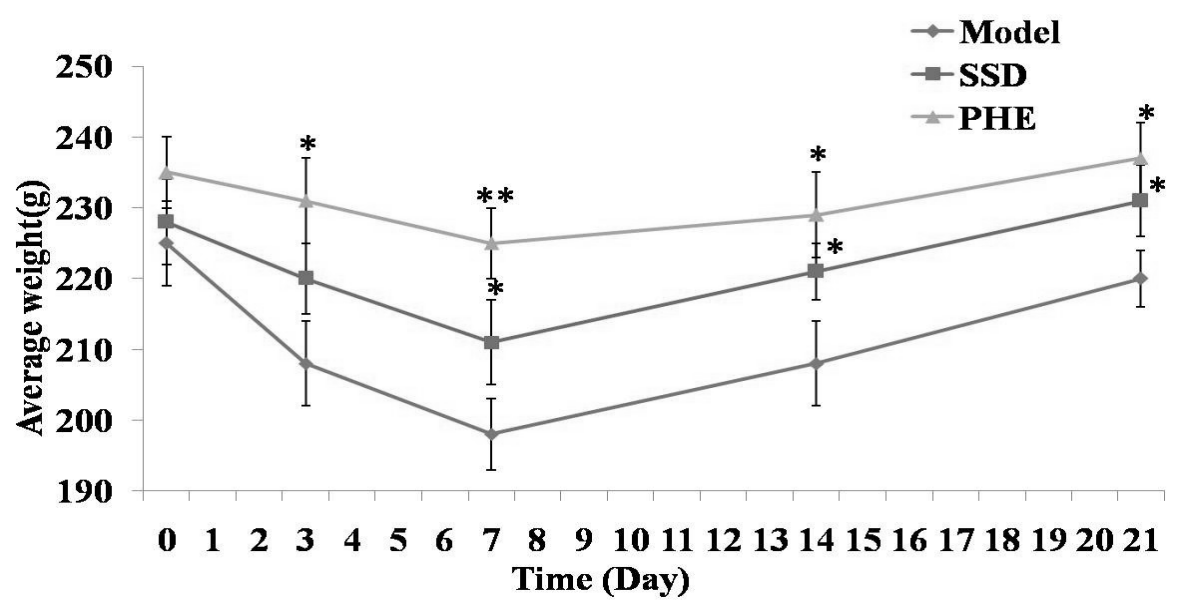

Figure 1: Body weight of rats as a function of healing time $(n=10)$. The data are expressed as mean \pm SEM; ${ }^{*} p$ $<0.05$ and ${ }^{* *} p<0.01$ were significantly different compared to control group 


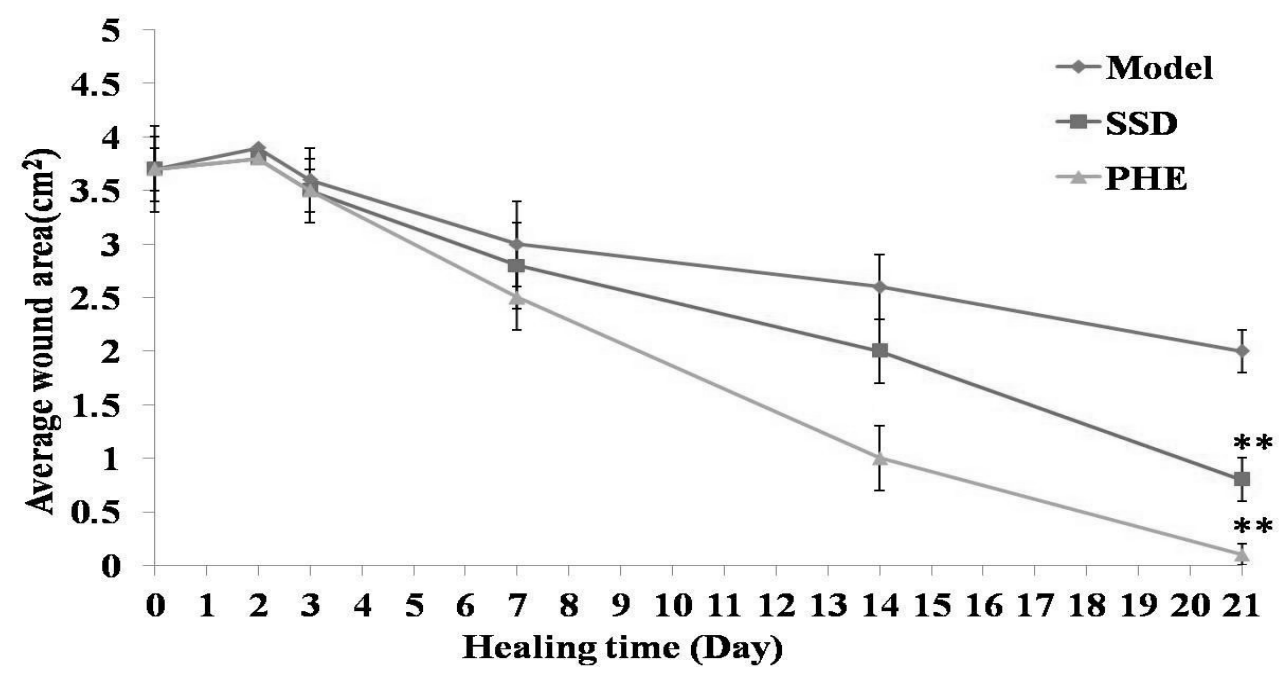

Figure 2: Evaluation of wound area as a function of time $(n=10)$. The data are expressed as mean \pm SEM; ${ }^{*} p<$ 0.05 and ${ }^{* *} p<0.01$ were considered significantly different compared to control group

\section{Histological features}

Histological findings on the wounded skin, treated with SSD and PHE on day 7,14 and 21 are shown in Plate 1. On day 7 , inflammatory cell infiltrated severely and some fibroblast and granulation tissues were found in the skin of model control rats. The skin burn of the rats treated with SSD or PHE showed reduced inflammatory cells and appearance of several fibroblasts and granulation tissues. On day 14, several inflammatory cells and some fibroblast and granulation tissues were found in skin burns of control rats. However, many new collagen fibers increased, inflammatory cell decreased heavily and a lot of fibroblast and granulation tissues appeared in the skin burn of rats treated with SSD or PHE.

On day 21 , the inflammatory cells were still seen and some fibroblast and granulation tissue grow in skin burn of model rats. However, there were fleshy buds constituted with blood vessels and fibroblasts, inflammatory cells disappeared and were replaced by new granulation tissue, and epithelialization progressed very quickly in rats treated with SSD or PHE. The results suggest that PHE treatment had a beneficial influence on the various phases of wound healing.

\section{Antibacterial activity}

The antibacterial results revealed that the MIC of PHE on $S$. aureus, $P$. aeruginosa and $E$. coli

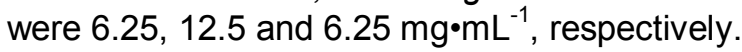

\section{DISCUSSION}

Thermal burn injury is still a major cause of death and disability in the world and its healing process is a challenge in modern medicine. Burns on the human body may be treated by different methods depending on the extent and severity of the burns. SSD can kill a wide variety of bacteria, so it is commonly used for preventing and treating infections of second and third degree burns. Recent studies revealed that SSD ointment has positive effects on proliferation of fibroblasts which are the main source of collagen and fibronectin [6]. However, current reports suggest that silver-based products have serious side effects [3] and researchers are making efforts to seek better topical antimicrobial products.

The weight loss is due to the severe metabolic, hormonal, immunologic and circulatory disturbances caused by the burns [7]. Up to day 7 , serum catecholamine, cortisol and glucagon and pro-inflammatory cytokines, in particular IL-8 levels increased significantly in burned rats $[8,9]$. These hormonal changes promote increased proteolysis with the release of high amounts of alanine and glutamine, glycerol and free fatty acids into the systemic circulation. The consequence of this hormonal reaction is an increase in the energy expenditure which can reach $100 \%$ in case of severe burns. Such levels of loss can persist $7-10$ days and even more, which explains that weight decreased immediately after injury [10].

In the treatment of burn, the committed step is to control bacterial infection. The common and main bacteria isolated from clinical burnt patients were $S$. aureus, $P$. aeruginosa and $E$. coli. SSD is the 
Day 7

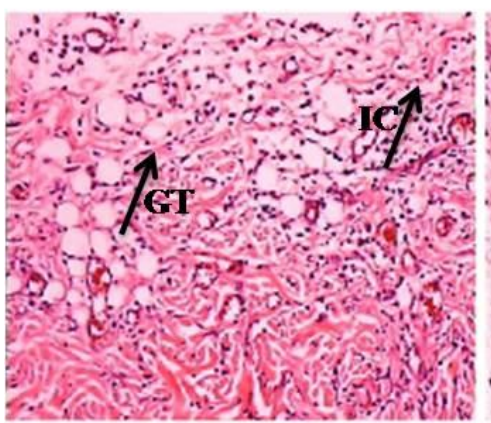

Day 14

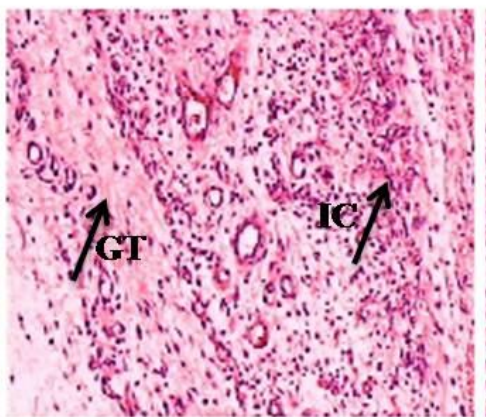

Day 21

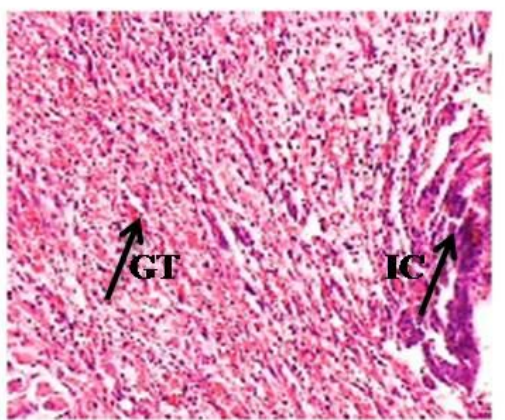

a
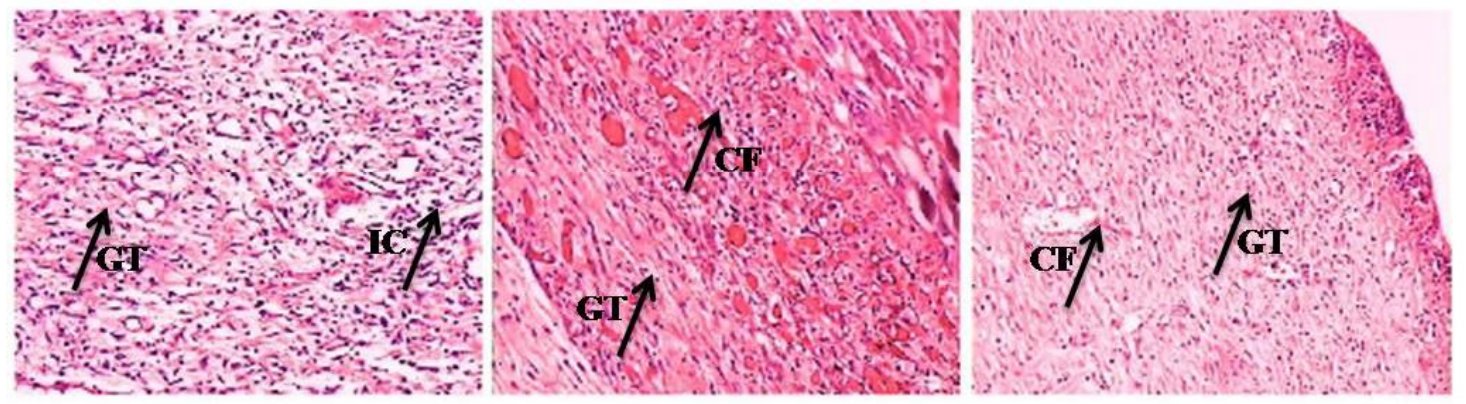

b
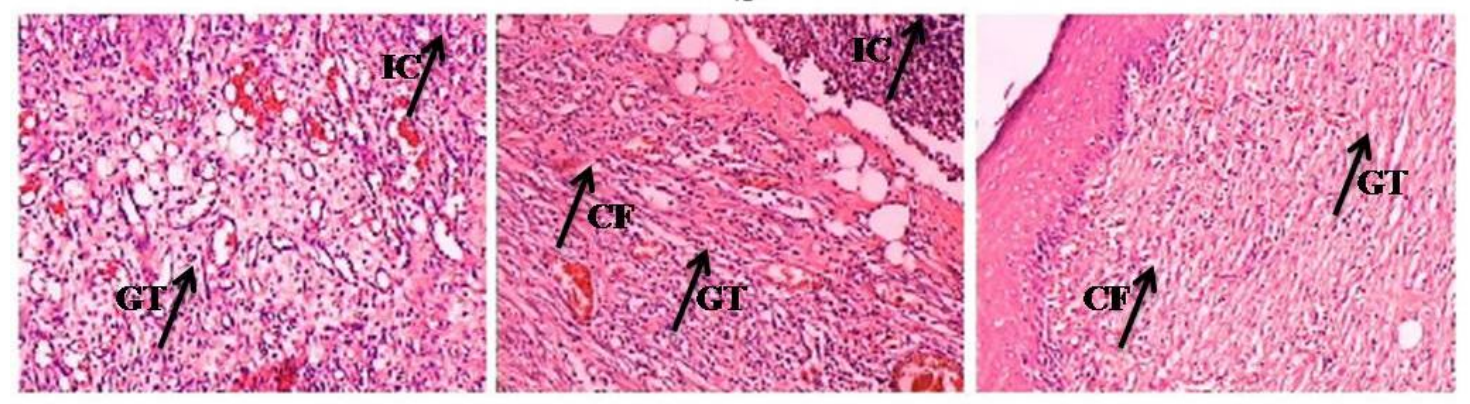

c

Plate 1: Plate 1: Photographs of pathological sections of burnt skin (a) Control, (b) SSD, and (c) PHE on days 7 , 14 and 21, respectively $(H \& E, \times 100)$; IC = inflammatory cell, $G T=$ granulation tissues, and $C F=$ collagen fibers

most used topical treatment for burn injury due to its anti-microbial efficacy. However, it has systemic complications such as neutropenia, methaemoglobinemia and renal toxicity[3].

Traditional Chinese herb has the advantage of inhibiting bacterial growth with few side effects. In our study, PHE inhibited the growth of bacteria effectively. Therefore, PHE could control bacterial infection in burn wound for better healing.

In recent years, there has been a growing interest in alternative medicines and natural medicinal products for the local treatment of wounds due to the high costs of traditional drug treatments [11]. Skin integrity is restored by a physiological process aimed at repairing the damaged tissues. The healing process proceeds in four phases: hemostasis, inflammation, proliferation and remodeling [12]. The time required for complete healing of deep second degree burns, without the application of specific therapeutic agents, can be three to six weeks or more and these burns will leave a scar tissue that may undergo hypertrophy and contract itself [13]. In our study, PHE accelerated the scab of deep degree burn wound and prevented infection effectively. The burn wound of model rats with PHE treatment healed well on day 21 while that of model rats still showed severe inflammatory cell infiltration without any treatment through histological analysis. 


\section{CONCLUSION}

This study reveals that PHE accelerates the healing of burn wounds and possesses strong anti-bacterial activity. Thus pomegranate husk is a promising herb for burns treatment.

\section{REFERENCES}

1. Nacer Khodja A, Mahlous M, Tahtat D. Evaluation of healing activity of PVA/chitosan hydrogels on deep second degree burn: pharmacological and toxicological tests. Burns 2013; 39: 98-104.

2. Edelman LS. Social and economic factors associated with the risk of burn injury. Burns 2007; 33: 958-965.

3. Shanmugasundaram N, Uma TS, Ramyaa TS, Lakshmi, Babu M. Efficiency of controlled topical delivery of silver sulfadiazine in infected burn wounds. J Biomed Mater Res A. 2008; 89: 472-473.

4. Seeram NP, Adams LS, Henning SM. In vitro antiproliferative, apoptotic and antioxidant activities of punicalagin, ellagic acid and a total pomegranate tannin extract are enhanced in combination with other polyphenols as found in pomegranate juice. J Nutri Bioc 2005; 16: 360-367.

5. European Commission. Directive 2010/63/EU on the protection of animals used for scientific purposes [cited 2013 Jan 16]. Available from:http://ec.europa.eu/environment/chemicals/lab_ animals/legislation_en.htm.

6. Coelho JM, Antoniolli AB, Nunes eSilva D. Effects of silver sulfadiazine, ipê roxo (tabebuia avellanedae) extract and barbatimão (Stryphnodendron adstringens) extract on cutaneous wound healing in rats. Rev. Col. Bras. Cir. 2010; 37: 45-51.

7. Wolfe RR. Relation of metabolic studies to clinical nutrition: the example of burn injury. Am J Clin Nutr 1996; 64: 800-808.

8. De Bandt JP, Chollet-Martin S, Hernvann A, Lioret N, Desroys Du Roure L, Lim SK. Cytokine response to burn injury: relationship with protein metabolism. $J$ Trauma 1994; 36: 624-628.

9. Wilmore DW. Hormonal responses and their effect on metabolism. Surg Clin North Am 1976; 56: 999-1018.

10. Bessey $P$, Jiang Z, Johnson D. Post traumatic skeletal muscle proteolysis: the role of the hormonal environment. World J Surg 1989; 13: 465-470.

11. Lee JA, Jeong $H J$, Park $H J$, Jeon $S$, Hong SU. Acupuncture accelerates wound healing in burnedinjured mice. Burns 2011; 37: 117-125.

12. Pazyar N, Yaghoobi R, Rafiee E, Mehrabian A, Feily A.Skin wound healing and phytomedicine: a review. Skin Pharmacol Physiol. 2014; 27(6): 303-310.

13. Johnson RM, Richard R. Partial-thickness burns identification and management. Adv Skin Wound Care 2003; 16: 178-187. 